\title{
RIESZ DECOMPOSITIONS FOR SCHRÖDINGER OPERATORS ON GRAPHS
}

\author{
FLORIAN FISCHER AND MATTHIAS KELLER
}

\begin{abstract}
We study superharmonic functions for Schrödinger operators on general weighted graphs. Specifically, we prove two decompositions which both go under the name Riesz decomposition in the literature. The first one decomposes a superharmonic function into a harmonic and a potential part. The second one decomposes a superharmonic function into a sum of superharmonic functions with certain upper bounds given by prescribed superharmonic functions. As application we show a Brelot type theorem.

Keywords: Potential theory, Green's function, Schrödinger operator, weighted graph, subcritical, greatest harmonic minorant.
\end{abstract}

\section{INTRODUCTION}

Schrödinger operators in the Euclidean space have been studied for a long time and a profound potential theory has been developed. On graphs potential theory was mainly studied in the context of random walks. However, in recent years there is a rising interest in general Schrödinger operators which goes beyond the probabilistic framework. The analysis and spectral theory of these operators received enormous attention, see e.g. [BGK15, BP18, CdVTHT11, GKS16, GMT14, GS11, KL12, KLSW17, KR16, KS17]. Especially, the study of Hardy inequalities [Gol14, KPP18] relies on a profound understanding of (super)harmonic functions, see also [Fit00, KPP17, Tak14, Tak16].

A classical and fundamental tool to study superharmonic functions are Riesz decompositions. In this paper we study two of these decompositions for superharmonic functions of Schrödinger operators on graphs. The first decompositions deals with superharmonic functions which are bounded from below by a (sub)harmonic function. Then, the superharmonic function can be decomposed into a harmonic and a potential part, see Theorem 2.4. Such a decomposition is referred to in the literature as Riesz decomposition, see [AG01, Hel73, Hel09]). The second decomposition considers a superharmonic function $s$ which is smaller than the sum of two superharmonic functions $s_{1}$ and $s_{2}$. Then, $s$ can be decomposed into the sum of two superharmonic functions $r_{1}$ and $r_{2}$ such that $r_{1} \leq s_{1}$ and $r_{2} \leq s_{2}$, see Theorem 2.8. In the literature this is also referred to as Riesz decompostion, see [BB87, Han87], but also as Mokobodzki-Sibony decomposition, see [Hel09], so, we will refer to it as Riesz-Mokobodzki-Sibony decomposition. 
In the context of random walks on graphs the first decomposition, the Riesz decompostion, is well known for non-negative superharmonic functions, see [KSK66, Soa94, Woe00, Woe09]. However, the Schrödinger operators we study here do not have a probabilistic interpretation. Moreover, we wish to treat not only nonnegative superharmonic functions but also superharmonic functions which are only bounded from below by a subharmonic functions. To this end, the probabilistic approach does not seem to work and we rely on potential theoretic arguments to obtain the result. However, one can recover a substantial part of the probabilistic method via the ground state transform. In this case we even get a probabilistic type representation of the harmonic part and an alternative formula for the potential part in the Riesz decomposition, see Theorem 5.1.

For the second decomposition, the Riesz-Mokobodzki-Sibony decomposition, we are not aware of a discrete analogue. Although it is certainly well known in the context of random walks, again our proof for Schrödinger operators relies on potential theoretic arguments rather than probabilistic ones. We expect this to be useful in the study of limits of superharmonic functions at the Martin boundary.

As an application we present a Brelot type theorem. In the continuum this theorem gives an equality for the charge of a superharmonic function in terms of the infimum of the quotient of the function and the Green's function. However, in contrast to the continuum setting we only get one inequality which we show to be strict.

The paper is structured as follows. In the next section, Section 2. we introduce the setting and present the main results. In Section 3, we study the fundamental tools to prove the main theorems such as the Dirichlet problem and greatest harmonic minorants. In Section 4 we prove the main theorems and in Section 5 we give a probabilistic type representation of the Riesz decompostion. Finally, in Section 6 we show a Brelot type theorem.

\section{Setting the Scene And Main Results}

In this section we present the underlying notions of this work and state the main results.

2.1. Graphs, Schrödinger Operators and Subcriticality. Let $X$ be an infinite set equipped with the discrete topology. Let a symmetric function $b: X \times X \rightarrow$ $[0, \infty)$ with zero diagonal be given such that $b$ is locally summable, i.e., the vertex degree deg satisfies

$$
\operatorname{deg}(x)=\sum_{y \in X} b(x, y)<\infty
$$

for all $x \in X$. We refer to $b$ as a graph over $X$ and the elements of $X$ are called vertices. A subset $W \subseteq X$ is called connected with respect to the graph $b$, if for every vertices $x, y \in W$ there is a path $x_{0}, \ldots, x_{n} \in W$, such that $x=x_{0}, y=x_{n}$ 
and $b\left(x_{i-1}, x_{i}\right)>0$ for all $i \in\{1, \ldots, n\}$. Throughout this paper we will always assume that

$X$ is connected with respect to the graph $b$.

The space of real valued functions on $W \subseteq X$ is denoted by $\mathcal{C}(W)$ and the space of functions with compact support in $W$ is denoted by $\mathcal{C}_{c}(W)$. We consider $\mathcal{C}(W)$ to be a subspace of $\mathcal{C}(X)$ by extending the functions of $\mathcal{C}(W)$ by zero on $X \backslash W$.

A strictly positive function $m: X \rightarrow(0, \infty)$ extends to a measure with full support via $m(W)=\sum_{x \in W} m(x)$ for $W \subseteq X$.

For $W \subseteq X$, let the space $\mathcal{F}(W)=\mathcal{F}_{b}(W)$ be given by

$$
\mathcal{F}(W)=\left\{f \in \mathcal{C}(X)\left|\sum_{y \in W} b(x, y)\right| f(y) \mid<\infty \text { for all } x \in X\right\} .
$$

We set $\mathcal{F}=\mathcal{F}(X)$ and define the (formal) Schrödinger operator $H=H_{b, c, m}$ on $\mathcal{F}$ via

$$
H f(x)=\frac{1}{m(x)} \sum_{y \in X} b(x, y)(f(x)-f(y))+\frac{c(x)}{m(x)} f(x), \quad x \in X,
$$

where $c \in \mathcal{C}(X)$ is a function. A function $u \in \mathcal{F}$ is harmonic, (superharmonic, subharmonic) on $W \subseteq X$ if

$$
H u=0 \quad(H u \geq 0, H u \leq 0) \text { on } W .
$$

The operator $H_{b, c, m}$ is said to be non-negative on $\mathcal{C}_{c}(X)$ if for all $\varphi \in \mathcal{C}_{c}(X)$ we have

$$
\sum_{x \in X}\left(H_{b, c, m} \varphi\right)(x) \varphi(x) m(x) \geq 0, \quad x \in X .
$$

By the Allegretto-Piepenbrink theorem, [HK11, KPP17], this is equivalent to the existence of a positive superharmonic function.

2.2. Green's Functions and Potentials. Let a graph $b$ and a function $c$ be given such that $H$ is non-negative. We denote the restriction of the operator $H$ to $\mathcal{C}(K)$ for a finite set $K$ by $H^{K}$. It is not hard to see that $H^{K}$ is invertible on $\mathcal{C}(K)$ due non-negativity of $H$ and the connectedness of $X$, confer [KPP17, Lemma 5.15]. Furthermore, due to domain monotonicity one has $\left(H^{K}\right)^{-1} \varphi \leq\left(H^{L}\right)^{-1} \varphi$ for $\varphi \in$ $\mathcal{C}_{c}(K)$ for $K \subseteq L$.

Let an increasing exhaustion $\left(K_{n}\right)$ of $X$ with finite sets be given. We define the function $G=G_{b, c, m}: X \times X \rightarrow[0, \infty]$ via

$$
G(x, y)=\lim _{n \rightarrow \infty}\left(H^{K_{n}}\right)^{-1} 1_{y}(x),
$$

for $x, y \in X$, where $1_{y}$ is the characteristic function at $y \in X$, confer [KPP17, Theorem 5.16].

Indeed, $G$ is independent of the choice of $\left(K_{n}\right)$. In general $G$ can take the value $\infty$ but if $G(x, y)<\infty$ holds for some $x, y \in X$, then it holds for all $x, y \in X$, 
see [KPP17, Theorem 5.12]. Moreover, if the Green's function is finite for some measure $m$ then it can be checked that it is finite for all measures, confer [Sch11].

We call $G=G_{b, c, m}$ the Green's function of $H=H_{b, c, m}$ in $X$. We call the operator $H$ subcritical if for some (all) $x, y \in X$ we have

$$
G(x, y)<\infty .
$$

Remark 2.1. In [KPP17, Theorem 5.3] it is shown that subcriticality is equivalent to the validity of a Hardy inequality, i.e., the existence of a function $w \geq 0$ on $X$ such that

$$
\sum_{x \in X}(H \varphi)(x) \varphi(x) m(x) \geq \sum_{x \in X} w(x) \varphi^{2}(x), \quad \varphi \in \mathcal{C}_{c}(X) .
$$

Moreover, subcriticality is equivalent to the existence of a least two linearly independent positive superharmonic functions and thence it implies that the corresponding Schrödinger operator is also non-negative.

In probability theory one considers graphs with $c \geq 0$ and the measure $m=$ $\operatorname{deg}+c$. In this context a graph with a subcritical Schrödinger form is called transient, see e.g. [Fit00, FOT10, KPP17, Soa94, Woe00, Woe09], for an elaborate study on transience.

Remark 2.2. There are equivalent formulations of the Green's function via resolvents or semigroups of a self-adjoint realization $H_{m}$ on $\ell^{2}(X, m)$ of the Schrödinger operator $H$, i.e.,

$$
G(x, y)=\lim _{\alpha \downarrow 0}\left(H_{m}+\alpha\right)^{-1} 1_{y}(x)=\int_{0}^{\infty} \mathrm{e}^{-t H_{m}} 1_{y}(x) \mathrm{d} t, \quad x, y \in X .
$$

Here, $\ell^{2}(X, m)$ denotes the space of square $m$-summable functions. For details see [KPP17].

The function $G$ is strictly positive, symmetric with respect to $m$, superharmonic and if $H$ is subcritical then for all $y \in X$

$$
H G(y, \cdot)=H G(\cdot, y)=1_{y} .
$$

Moreover, for fixed $y \in X$, the function $G(\cdot, y)$ is the smallest function $u \geq 0$ in $\mathcal{F}$ such that $H u \geq 1_{y}$, see [KPP17, Theorem 5.16].

We denote the space of $G$-integrable functions on $X$ by $\mathcal{G}=\mathcal{G}_{b, c, m}$

$$
\mathcal{G}=\left\{f \in \mathcal{C}(X)\left|\sum_{y \in X} G(x, y)\right| f(y) \mid<\infty \text { for all } x \in X\right\} .
$$

Clearly, $\mathcal{G}$ is non-empty if and only if $H$ is subcritical in which case it obviously includes $\mathcal{C}_{c}(X)$. For $f \in \mathcal{G}$ and $x \in X$, we denote

$$
G f(x)=\sum_{y \in X} G(x, y) f(y) .
$$

Decomposing $f \in \mathcal{G}$ into positive and negative parts and approximating these parts monotonously via compactly supported functions we have $G f \in \mathcal{F}$ and

$$
H G f=f
$$


by monotone convergence.

Definition 2.3. Let $H=H_{b, c, m}$ be subcritical. A function $p \in \mathcal{C}(X)$ is called a potential if there is $f \in \mathcal{G}$ such that

$$
p=G f .
$$

The function $f$ is then called a charge of $p$ and $p$ the potential of $f$.

2.3. Main Results. In this subsection we present the main results of the paper which are two decompositions for superharmonic functions. Both are known as Riesz decompositions in the literature.

The first decomposition allows us to decompose a superharmonic function into a harmonic and a potential part (provided the superharmonic function is bounded from below by a subharmonic function). Recall that a function is called a minorant of another function if it is smaller or equal everywhere.

The second decomposition, which is also known as the Mokobodzki-Sibony decomposition, states that if a the sum of two positive superharmonic functions has a positive superharmonic minorant, then this minorant can be decomposed into two positive superharmonic minorants of the original superharmonic functions.

Recall that a function $g \in \mathcal{C}(X)$ is called minorant of $f \in \mathcal{C}(X)$ if $g \leq f$. Moreover, $h \in \mathcal{C}(X)$ is called greatest harmonic minorant of $f \in \mathcal{C}(X)$ if $h$ is a harmonic minorant of $f$ and for all other harmonic minorants $g$ of $f$ we have $g \leq h$. Clearly, the greatest harmonic minorant is unique in case it exists and we write

$$
h=\operatorname{ghm}_{f} .
$$

Next, we present our first main result, the Riesz decomposition.

Theorem 2.4 (Riesz Decomposition). Let $H=H_{b, c, m}$ be subcritical and let $s$ be a superharmonic function with a subharmonic minorant. Then there exists a unique decomposition

$$
s=s_{p}+s_{h}
$$

such that $s_{p}$ is a non-negative potential with charge $H s \in \mathcal{G}$ and $s_{h}=\operatorname{ghm}_{s}$, in particular, $s$ has a greatest harmonic minorant. Moreover, if $s \geq 0$ then $s_{h} \geq 0$, and $s$ is the potential of a non-negative charge if and only if $s_{h}=0$.

Remark 2.5. As stated in the theorem the potential part is given by $s_{p}=G H s$. Furthermore, it becomes clear from the proof that the harmonic part arises as $s_{h}=\lim _{n \rightarrow \infty} s_{n}$, where $s_{n}$ is the solution of the Dirichlet problem with respect to $s$ on an increasing exhaustion $\left(K_{n}\right)$ of $X$ with finite sets, see Theorem 3.4.

Remark 2.6. For a continuous analogue of Theorem 2.4, see [AG01, Theorem 4.4.1] or also [Hel09, Theorem 3.5.11]. In these works one proves the decomposition by approximating the charge of the potential part by compactly supported functions and taking the monotone limit. We use a similar strategy to prove the decomposition in the discrete case. The continuous version of this theorem goes back to 
[Rie30, p. 350]. There is also a discrete version of this theorem in the context of transient random walks, i.e., $c \geq 0$, see e.g. [Woe09, Theorem 6.43], [Woe00, Theorem 24.2], [Soa94, Theorem 1.38]. There, the superharmonic function is assumed to be non-negative.

Remark 2.7. Let us comment on a related decomposition in the literature. In [Soa94] (see also $\left[\mathrm{GHK}^{+} 15\right]$ ) the so called Royden decomposition is proven for functions of finite energy. It states that such a function can be decomposed into a function which can be approximated by compactly supported functions and a harmonic functions. The proof relies on the Hilbert space structure of the functions of finite energy which we do not have at our disposal in our situation.

Our second main result is the so-called Riesz-Mokobodzki-Sibony Decomposition.

Theorem 2.8 (Riesz-Mokobodzki-Sibony Decomposition). Let $H_{b, c, m}$ be subcrit$i$ cal and let $s, s_{1}, s_{2} \geq 0$ be superharmonic functions such that $s \leq s_{1}+s_{2}$. Then there exist unique superharmonic functions $0 \leq r_{1} \leq s_{1}$ and $0 \leq r_{2} \leq s_{2}$ such that

$$
s=r_{1}+r_{2} \text {. }
$$

For the continuous analogue, see [Hel09, Theorem 4.6.9].

Remark 2.9. The name of the Riesz-Mokobodzki-Sibony decomposition seems to have changed over the years. In [BB87] and [Han87] the continuous version of this theorem is called Riesz decomposition. In [Han87] this decomposition is also one of the four assumptions of a so-called balayage space. In [Hel09] the name Mokobodzki-Sibony theorem is used. The first proof of such a theorem in the continuum seems to go back to [MS68].

Remark 2.10. These Riesz decompositions are the fundamental tools to develop a Choquet-Martin boundary theory which generalises the existing theory in the probabilistic case, see [Woe00], to all graphs with corresponding subcritical Schrödinger operator, see [Fis18, Chapter 5]. For instance, Theorem 2.4 is the crucial step to get the so-called discrete Poisson-Martin integral representation. To gain certain boundary limits, Theorem 2.8 is of peculiar interest, see [Fis18, Section 4.4 and Chapter 6].

\section{ToOlbox}

The proofs of the theorems above are inspired by classical potential theoretic arguments in the continuum case as they can be found [AG01] and [Hel09]. To this end, solutions of a Dirichlet problems along an exhaustion play a crucial role.

For the remainder of the section let $b$ be a connected graph and let $c$ be a function such that the operator $H=H_{b, c, m}$ is non-negative. 
3.1. Dirichlet Problems on Finite Subgraphs. The Dirichlet problem on $W \subseteq$ $X$ with respect to $f \in \mathcal{C}(X)$ is the problem of finding a function $u \in \mathcal{C}(X)$ such that

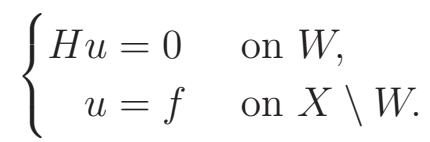

The function $u \in \mathcal{C}(X)$ is then referred to as the solution of the Dirichlet problem on $W$ with respect to $f$.

It is well known that due to positivity of $H$ and connectedness of the graph these Dirichlet problems always have a unique solution. For the convenience of the reader we provide a short argument.

Lemma 3.1 (Existence of Unique Solutions to Dirichlet Problems). Let $H_{b, c, m}$ be non-negative on $\mathcal{C}_{c}(X)$, let $K \subseteq X$ be finite and let $f \in \mathcal{F}$. Then there exists a unique solution $u$ to the Dirichlet problem on $K$ with respect to $f$. Moreover, if $f \geq 0$, then $u \geq 0$.

Proof. By a direct calculation, one sees that $u$ is a solution to the Dirichlet problem if and only if $u$ satisfies

$$
H_{b, c+d, m} u=g \quad \text { on } K,
$$

where $d(x)=\sum_{y \in X \backslash K} b(x, y)$ and $g(x)=\frac{1}{m(x)} \sum_{y \in X \backslash K} b(x, y) f(y)$ for $x \in K$ and $d=g=0$ on $X \backslash K$. Note that the sum in the definition of $g$ converges absolutely due to the assumption $f \in \mathcal{F}$. Since the restriction $H_{b, c+d, m}^{K}$ is invertible on $\mathcal{C}_{c}(K)$, confer [KPP17, Lemma 5.15], and the resolvent is positivity preserving, confer [KPP17, Corollary 3.5], we obtain the result.

An important tool for the following potential theory on graphs is the so-called minimum principle.

Theorem 3.2 (Minimum Principle, Lemma 5.14 in [KPP17]). Let $H=H_{b, c, m}$ be non-negative on $\mathcal{C}_{c}(X)$. If $u \in \mathcal{F}$ satisfies $(H+\alpha) u \geq 0$ for $\alpha \geq 0$ on a finite set $K \subseteq X$ and $u \geq 0$ on $X \backslash K$, then $u=0$ or $u>0$ on $K$.

The minimum principle has the following immediate corollary.

Corollary 3.3. Let $H_{b, c, m}$ be non-negative and let $s$ be a superharmonic function. Then for any finite set $K$ the unique solution $u$ to the Dirichlet problem on $K$ with respect to $s$ satisfies

$$
u \leq s
$$

Proof. Let $u$ be the unique solution of the Dirichlet problem on a finite set $K$ with respect to $s$. On $K$, we have $H(s-u) \geq 0$ and on $X \backslash K$ we have $s-u=0$. By the minimum principle, Theorem 3.2, we get that $s \geq u$ on $K$. 
3.2. Existence and Properties of Greatest Harmonic Minorants. In this section we study greatest harmonic minorants of superharmonic functions. Specifically, we show that these greatest harmonic minorants exist whenever there is a subharmonic minorant. Moreover, we prove that greatest harmonic minorants can be approximated by solutions of Dirichlet problems, are additive and monotone. Later we show that the greatest harmonic minorant of the Green's function is the zero function. These results are well known in classical potential theory in the continuum, see [Hel73, Section 3.3], but they seem to be new in the setting of Schrödinger operators on graphs.

Theorem 3.4 (Existence and Properties of Greatest Harmonic Minorants). Let $H=H_{b, c, m}$ be non-negative on $\mathcal{C}_{c}(X)$. Let $s$ be superharmonic with subharmonic minorant $u$. Then s has a greatest harmonic minorant $\mathrm{ghm}_{s}$ such that $\mathrm{ghm}_{s} \geq u$.

Moreover, we have

$$
\operatorname{ghm}_{s}=\lim _{n \rightarrow \infty} s_{n}
$$

as a pointwise limit, where $s_{n}$ is the solution of the Dirichlet problem with respect to $s$ on an increasing exhaustion $\left(K_{n}\right)$ of $X$ with finite sets.

Furthermore, greatest harmonic minorants are additive, i.e., if $t$ is superharmonic with a subharmonic minorant, then ghm $_{s+t}$ exists and

$$
\operatorname{ghm}_{s+t}=\operatorname{ghm}_{s}+\operatorname{ghm}_{t} .
$$

Moreover, greatest harmonic minorants are monotone, i.e., if $s \leq t$, then

$$
\operatorname{ghm}_{s} \leq \mathrm{ghm}_{t}
$$

Proof. Let $\left(K_{n}\right)$ be an increasing exhaustion of $X$ with finite sets and let $s_{n}$ denote the solution of the Dirichlet problem on $K_{n}$ with respect to $s$ which exists for every $n \in \mathbb{N}$ by Lemma 3.1.

Firstly, we show the existence of the greatest harmonic minorant: By Corollary 3.3, we get $s \geq s_{n}$ on $K_{n}$ for every $n \in \mathbb{N}$. On $K_{n}$, we have $H\left(s_{n}-s_{n+1}\right)=0$ and on $X \backslash K_{n}$, we have $s_{n}-s_{n+1}=s-s_{n+1} \geq 0$. Hence, by the minimum principle, Theorem 3.2, we get

$$
s_{n} \geq s_{n+1} \text { on } X
$$

for every $n \in \mathbb{N}$ which means that we have an decreasing sequence of harmonic functions. Thus, there exists a pointwise limit

$$
s_{\infty}=\lim _{n \rightarrow \infty} s_{n}
$$

which might take the value $-\infty$ on vertices. However, this is not the case: By assumption $s$ has a subharmonic minorant which we denote by $u$. Then, we have $H\left(s_{n}-u\right) \geq 0$ on $K_{n}$ and $s_{n}-u=s-u \geq 0$ on $X \backslash K_{n}, n \in \mathbb{N}$. By the minimum principle, Theorem 3.2, we obtain $s_{n} \geq u$ on $X$ for all $n \in \mathbb{N}$. So,

$$
s \geq s_{\infty} \geq u
$$


which shows finiteness of $s_{\infty}$. By monotone convergence, we find that the function $s_{\infty}$ is in $\mathcal{F}$ and

$$
0=\lim _{k \rightarrow \infty} H s_{k}=H s_{\infty}
$$

Thus, $s_{\infty}$ is a harmonic minorant.

Finally, let $v$ be another harmonic minorant. Then again, on $K_{n}$, we have $H\left(s_{n}-v\right)=0$ and $s_{n}-v=s-v \geq 0$ on $X \backslash K_{n}$. Hence, the minimum principle, Theorem 3.2, yields $s_{n} \geq v$ and, therefore, $s_{\infty} \geq v$. Thus,

$$
s_{\infty}=\operatorname{ghm}_{s} .
$$

Secondly, we show the additivity property: Consider the solutions of the Dirichlet problem $s_{n}$ and $t_{n}$ on $K_{n}$ with respect to $s$ and $t, n \in \mathbb{N}$. Since $s_{n}+t_{n}$ solves the Dirichlet problem

$$
\left\{\begin{aligned}
H w & =0 & & \text { on } K_{n}, \\
w & =s+t & & \text { on } X \backslash K_{n},
\end{aligned}\right.
$$

we obtain by the above

$\operatorname{ghm}_{s+t}=(s+t)_{\infty}=\lim _{n \rightarrow \infty}\left(s_{n}+t_{n}\right)=\lim _{n \rightarrow \infty} s_{n}+\lim _{n \rightarrow \infty} t_{n}=s_{\infty}+t_{\infty}=\operatorname{ghm}_{s}+\operatorname{ghm}_{t}$.

Thirdly, we show monotonicity: If $s \leq t$, then $\operatorname{ghm}_{s}$ is a harmonic minorant of $t$. Thus, $\operatorname{ghm}_{s} \leq \operatorname{ghm}_{t}$ as $\operatorname{ghm}_{t}$ is the greatest harmonic minorant of $t$.

3.3. Greatest harmonic minorants of potentials. Next, we relate greatest harmonic minorants and potentials. Recall that if $H_{b, c, m}$ is subcritical, then it is positive by the Allegretto-Piepenbrink theorem, [KPP17, Theorem 4.2] (see Remark 2.1 as well). Moreover, the Green's function exists in this case.

Theorem 3.5. Let $H_{b, c, m}$ be subcritical. Then, for $f \in \mathcal{G}$,

(a) $\operatorname{ghm}_{G f}$ exists,

(b) $\operatorname{ghm}_{G f} \leq 0$,

(c) $\operatorname{ghm}_{G f}=0$ if $f \geq 0$.

For the proof of this theorem we need a local Harnack inequality which is well known in the context of graphs see [KPP17, Theorem 4.5] as well as [HK11] and references therein.

Proposition 3.6 (Harnack Inequality, Theorem 4.5 in [KPP17]). Let $H_{b, c, m}$ be non-negative, let $K \subseteq X$ be a connected and finite set and let $f \in \mathcal{C}(X)$. Then there exits a constant $C=C(f)>0$ such that the such that for any $u \in \mathcal{F}, u \geq 0$, such that $(H-f) u \geq 0$ on $K$ we have

$$
\max _{K} u \leq C \min _{K} u .
$$

The constant $C(f)$ can be chosen monotonously in the sense that if $f \leq g$ then $C(f) \geq C(g)$. 
We recall from the discussion in Section 3.5 that $G(\cdot, y)$ is the smallest $v \geq 0$ such that $H v=1_{y}, y \in X$.

Proof of Theorem 3.5. For a function $f$, we let $f=f_{+}-f_{-}$, where $f_{ \pm}=\max \{ \pm f, 0\}$.

Ad (a): Let $f \in \mathcal{G}$ be given. Then, the function $-G f_{-}$is a subharmonic minorant to $G f$ since $G f=G f_{+}-G f_{-} \geq-G f_{-}$and $H\left(-G f_{-}\right)=-f_{-} \leq 0$. Hence, the greatest harmonic minorant of $G f$ exists by Theorem 3.4.

Ad (b) for $f \in \mathcal{C}_{c}(X)$ : We show the statement for $f=1_{y}, y \in X$, first and prove the statement for $f \in \mathcal{C}_{c}(X)$ afterwards. The statement for general $f \in \mathcal{G}$ is then proven after we have shown (c).

Let $y \in X$. We note that 0 is a harmonic minorant of the positive superharmonic Green's function $G(\cdot, y)$ and let $u$ be an arbitrary harmonic minorant of $G(\cdot, y)$. Then $H(G(\cdot, y)-u)=1_{y}$. Since $G(\cdot, y)$ is the smallest solution $v \geq 0$ to $H v=1_{y}$, it follows that $G(\cdot, y)-u \geq G(\cdot, y)$. Hence, $u \leq 0$ and $\operatorname{ghm}_{G(\cdot, y)}=0$.

Now, let $K$ be a connected and finite set such that $\operatorname{supp}(f) \subseteq K$. Since the functions $G(x, \cdot)$ and $G(\cdot, x)$ are positive and superharmonic for all $x, y \in K$ and $K$ is finite, we obtain by the Harnack inequality, Proposition 3.6, the existence of a constant $C>0$ such that

$$
G(x, z) \leq C \cdot G(x, y)
$$

for all $x, y, z \in K$. This implies for $x, y \in K$

$$
G f(x) \leq \max _{z \in K}|f(z)| \sum_{z \in K} G(x, z) \leq C \cdot \# K \cdot \max _{z \in K}|f(z)| G(x, y) .
$$

Let $u$ be the harmonic minorant of $G f$. Then, $u$ is a harmonic minorant of $G(\cdot, y)$ and, therefore, $u \leq \operatorname{ghm}_{G(\cdot, y)}$. But above we have shown $\operatorname{ghm}_{G(\cdot, y)}=0$.

Ad (c): Since $f \geq 0$, we have $G f \geq 0$ and $G f$ is superharmonic. Therefore, $\mathrm{ghm}_{G f}$ exists as 0 is a harmonic minorant for $G f$. Let $\left(K_{n}\right)$ be an increasing exhaustion of $X$ with finite sets and define $f_{n}=1_{K_{n}} f, n \in \mathbb{N}$. By (b), for $\mathcal{C}_{c}(X)$, we know that $\operatorname{ghm}_{G f_{n}} \leq 0$ and since 0 is a harmonic minorant we have $\operatorname{ghm}_{G f_{n}}=0$. Since $G f, G f_{n}, G\left(f-f_{n}\right)$ are non-negative superharmonic functions and $G f=G\left(f-f_{n}\right)+G f_{n}$, we can use the the additivity of greatest harmonic minorants to get

$$
0 \leq \operatorname{ghm}_{G f}=\operatorname{ghm}_{G\left(f-f_{n}\right)}+\operatorname{ghm}_{G f_{n}}=\operatorname{ghm}_{G\left(f-f_{n}\right)} \leq G\left(f-f_{n}\right)
$$

By monotone convergence, $G\left(f-f_{n}\right) \rightarrow 0$ as $n \rightarrow \infty$. So, we conclude that $\operatorname{ghm}_{G f}=0$.

Ad (b) for general $f \in \mathcal{G}$ : Let $f \in \mathcal{G}$. By (a) the greatest harmonic minorants of $G f_{+}$and $G\left(-f_{-}\right)=-G f_{-}$exist and by (c) we have $\operatorname{ghm}_{G f_{+}}=0$. By additivity of the greatest harmonic minorants, Theorem 3.4, we have

$$
\operatorname{ghm}_{G f}=\operatorname{ghm}_{G f_{+}}+\operatorname{ghm}_{-G f_{-}}=\operatorname{ghm}_{-G f_{-}} \leq-G f_{-} \leq 0 .
$$

This finishes the proof. 
Theorem 3.5 states that any potential has a greatest harmonic minorant. Recall that the Riesz decomposition theorem, Theorem 2.4, says that any superharmonic function with subharmonic minorant can be decomposed into a potential part with non-negative charge and a harmonic part. Hence, the greatest harmonic minorant of this potential part is the zero function.

\section{Proofs of the Main Results}

4.1. Proof of the Riesz Decomposition. We next prove one of the main theorems, the Riesz decomposition, Theorem 2.4.

Proof of Theorem 2.4. Firstly, we assume that $H s \in \mathcal{G}$ (and we show below that this is always the case). Then $G H s$ is a non-negative superharmonic function and we can apply $H$ to it. We show that

$$
u=s-G H s
$$

is the greatest harmonic minorant of $s$. We have $H u=H(s-G H s)=0$, so $u$ is a harmonic function. Moreover, by Theorem 3.5, we have $\operatorname{ghm}_{G H s}=0$. Thus, using additivity of the greatest harmonic minorants we get

$$
u=\operatorname{ghm}_{u}=\operatorname{ghm}_{u}+\operatorname{ghm}_{G H s}=\operatorname{ghm}_{u+G H s}=\operatorname{ghm}_{s} .
$$

Hence, $s_{h}=(s-G H s)$ and $s_{p}=G H s$, which shows the existence of the decomposition.

As for the uniqueness, let $s=G f+h$ be another decomposition with $f \in \mathcal{G}$ and $h$ harmonic. Then, $\operatorname{ghm}_{s}-h=G(f-H s)$ is harmonic and therefore,

$$
0=H\left(\mathrm{ghm}_{s}-h\right)=H G(f-H s)=f-H s .
$$

We infer $f=H s$ which readily implies $h=\operatorname{ghm}_{s}$.

Furthermore, $s \geq 0$ implies $\operatorname{ghm}_{s} \geq 0$ since 0 is a harmonic minorant in this case. Moreover, by Theorem 3.5 we get that $s$ is the potential of a non-negative charge if and only if $\operatorname{ghm}_{s}=0$ and the theorem for $H s \in \mathcal{G}$ is proven.

To finish the proof we show that $H s \in \mathcal{G}$. The idea is to find an upper bound for $G H s$. Let $\left(K_{n}\right)$ be an increasing exhaustion of $X$ with finite sets. Since we assumed that $s$ has a subharmonic minorant, ghm $_{s}$ exists by Theorem 3.4. Consider the function

$$
v_{n}=s-\operatorname{ghm}_{s}-G\left(1_{K_{n}} H s\right)
$$

for $n \in \mathbb{N}$. Since

$$
H v_{n}=H s-1_{K_{n}} H s \geq 0,
$$

and $v_{n}$ has a subharmonic minorant with $-G\left(1_{K_{n}} H s\right)$, the greatest harmonic minorant ghm $_{v_{n}}$ exists by Theorem 3.4 and

$$
-\operatorname{ghm}_{v_{n}} \leq G\left(1_{K_{n}} H s\right) .
$$


Since the greatest harmonic minorant of a potential is non-positive, Theorem 3.5, we have $-\operatorname{ghm}_{v_{n}} \leq 0$ and, therefore, ghm $_{v_{n}} \geq 0$. Thus, we obtain

$$
G\left(1_{K_{n}} H s\right)=s-\operatorname{ghm}_{s}-v_{n} \leq s-\operatorname{ghm}_{s}-\operatorname{ghm}_{v_{n}} \leq s-\operatorname{ghm}_{s} .
$$

Monotone convergence yields $G H s \leq s-\mathrm{ghm}_{s}$ and, therefore, $H s \in \mathcal{G}$. This finishes the proof.

4.2. Proof of the Riesz-Mokobodzki-Sibony Decomposition. Next, we prove the Riesz-Mokobodzki-Sibony decomposition. It states that if positive superharmonic functions $s, s_{1}, s_{2}$ satisfy $s \leq s_{1}+s_{2}$, then $s=r_{1}+r_{2}$ with superharmonic functions $0 \leq r_{1} \leq s_{1}$ and $0 \leq r_{2} \leq s_{2}$. The proof is inspired by the one of [Hel09, Theorem 4.6.9] in the continuum setting of $\mathbb{R}^{n}$.

We start the proof with two simple observations which will be used in the proof. The first lemma, Lemma 4.1, uses the existence of a strictly positive superharmonic function which follows directly from the Allegretto-Piepenbrink theorem, see [KPP17, Theorem 4.2].

Lemma 4.1. Let $H=H_{b, c, m}$ be non-negative on $\mathcal{C}_{c}(X)$. Then $\operatorname{deg}+c>0$.

Proof. By the Allegretto-Piepenbrink theorem there exists a strictly positive superharmonic function $s$. Then $H s \geq 0$ implies for all $x \in X$ that

$$
(\operatorname{deg}(x)+c(x)) s(x) \geq \sum_{y \in X} b(x, y) s(y)>0
$$

by the strict positivity of $s$.

The next lemma is well known, but we include the short proof for the convenience of the reader.

Lemma 4.2. Let $H=H_{b, c, m}$ be non-negative on $\mathcal{C}_{c}(X)$. Let $T$ be a subset of the set of non-negative superharmonic functions. Then, the function

$$
r(x)=\inf _{t \in T} t(x), \quad x \in X,
$$

is a non-negative superharmonic function.

Proof. Let $t \in T$ be fixed and $r$ as stated above. It is obvious that $r \geq 0$ and $r \in \mathcal{F}$. So, it remains to show that $H r \geq 0$. By Lemma 4.1 we have $\operatorname{deg}+c>0$ and $H t \geq 0$ implies

$$
t(x) \geq \frac{1}{\operatorname{deg}(x)+c(x)} \sum_{y \in X} b(x, y) t(y) \geq \frac{1}{\operatorname{deg}(x)+c(x)} \sum_{y \in X} b(x, y) r(y)
$$

for all $x \in X$. Taking the infimum over all $t \in T$ on the right-hand side yields $H r \geq 0$. 
Proof of Theorem 2.8. We first show existence and then uniqueness.

Existence: We define

$$
\mathcal{S}_{1}=\left\{v \geq 0: v \text { is superharmonic and } s-s_{2} \leq v\right\} \quad \text { and } \quad r_{1}=\inf \mathcal{S}_{1},
$$

as well as

$$
\mathcal{S}_{2}=\left\{v \geq 0: v \text { is superharmonic and } s-r_{1} \leq v\right\} \quad \text { and } \quad r_{2}=\inf \mathcal{S}_{2},
$$

where the infimums are taken pointwise. By Lemma 4.2, the functions $r_{1}$ and $r_{2}$ are non-negative superharmonic and, thus, $r_{1} \in \mathcal{S}_{1}$ and $r_{2} \in \mathcal{S}_{2}$.

We show $s=r_{1}+r_{2}$ by proving several claims.

Claim 1. We have $s \leq r_{1}+r_{2}$.

Proof of the claim. This follows directly from $r_{2} \in \mathcal{S}_{2}$.

It remains to show that $s \geq r_{1}+r_{2}$. This is done in several steps.

Claim 2. If $s-r_{2}$ is superharmonic, then $s \geq r_{1}+r_{2}$.

Proof of the claim. Since $s \in \mathcal{S}_{2}$, we obtain

$$
s-r_{2} \geq 0 \text {. }
$$

On the other hand since $r_{1} \in \mathcal{S}_{1}$ we have $s-s_{2} \leq r_{1}$ and, therefore, $s-r_{1} \leq s_{2}$ which yields $s_{2} \in \mathcal{S}_{2}$. We obtain $r_{2} \leq s_{2}$ and therefore,

$$
s-s_{2} \leq s-r_{2}
$$

So, if $s-r_{2}$ is superharmonic, then $s-r_{2} \in \mathcal{S}_{1}$. As a consequence, $s-r_{2} \geq r_{1}$ which yields

$$
s \geq r_{1}+r_{2} .
$$

This proves the claim.

To prove that $s-r_{2}$ is superharmonic we need the following notation. Recall that $\operatorname{deg}+q>0$ by Lemma 4.1. For $f \in \mathcal{F}$ and $x \in X$, the function

$$
f_{x}(z)= \begin{cases}\frac{1}{\operatorname{deg}(x)+c(x)} \sum_{y \in X} b(x, y) f(y) & : z=x \\ f(z) & : z \neq x\end{cases}
$$

solves Dirichlet problem on $\{x\}$ with respect to $f$ which can be seen by the following direct calculation

$$
H f_{x}(x)=(\operatorname{deg}(x)+c(x)) f_{x}(x)-\sum_{x \in X} b(x, y) f_{x}(y)=0, \quad x \in X .
$$

For what follows, we write $r_{1, x}=\left(r_{1}\right)_{x}$ and $r_{2, x}=\left(r_{2}\right)_{x}$.

Claim 3. We have $r_{2}-r_{2, x} \leq s-s_{x}$.

Proof of the claim. Consider the following auxiliary function

$$
w=\min \left\{r_{2}, r_{2, x}+s-s_{x}\right\} .
$$


Note that $w=r_{2}$ on $X \backslash\{x\}$ as $r_{2}=r_{2, x}$ and $s=s_{x}$ outside of $x$. Clearly, $w \leq r_{2}$. So if we show that $w \in \mathcal{S}_{2}$, then $w=r_{2}$ and the claim follows. First of all, since the minimum of superharmonic functions is superharmonic, Lemma 4.2, we have $H w \geq 0$. Furthermore, $w \geq 0$ since $r_{2} \geq r_{2, x} \geq 0$ and $s \geq s_{x}$ by Lemma 3.1 and Corollary 3.3. We are left to show that $s-r_{1} \leq w$. On the one hand, $s-r_{1} \leq r_{2}$ by Claim 1. Moreover, this inequality $s \leq r_{1}+r_{2}$, implies by Lemma 3.1

$$
s_{x} \leq\left(r_{1}+r_{2}\right)_{x}=r_{1, x}+r_{2, x} .
$$

Now, combining this inequality with $r_{1} \geq r_{1, x}$, Corollary 3.3, we get

$$
r_{1}+r_{2, x}+s-s_{x} \geq r_{1, x}+r_{2, x}+s-s_{x} \geq s .
$$

Thus, we have $s-r_{1} \leq w$ which finishes the proof of the claim.

Claim 4: The function $s-r_{2}$ is superharmonic.

Proof of the claim. By Claim 3, $r_{2}-r_{2, x}-s_{x} \leq s$ for all $x \in X$ and, therefore,

$s(x)-r_{2}(x) \geq s_{x}(x)-r_{2, x}(x)=\left(s-r_{2}\right)_{x}(x)=\frac{1}{\operatorname{deg}(x)+c(x)} \sum_{y \in X} b(x, y)\left(s-r_{2}\right)(y)$.

This shows that $s-r_{2}$ is superharmonic in $x$. Applying this argument to every $x \in X$ we get that $s-r_{2}$ is superharmonic on $X$.

In summary Claim 1 shows $s \leq r_{1}+r_{2}$ and Claim 4 combined with Claim 2 yields $s \geq r_{1}+r_{2}$. Hence, $s=r_{1}+r_{2}$ and the proof of existence is finished.

We finally turn to uniqueness.

Uniqueness: Assume that there are non-negative superharmonic functions $t_{1}$ and $t_{2}$ for which $t_{1} \leq s_{1}, t_{2} \leq s_{2}$ and $s=t_{1}+t_{2}$. Then $s \leq t_{1}+s_{2}$ and $s \leq s_{1}+t_{2}$. Hence, $t_{1} \in \mathcal{S}_{1}$ and $t_{2} \in \mathcal{S}_{2}$ which readily gives $r_{1} \leq t_{1}$ and $r_{2} \leq t_{2}$. Since $r_{1}+r_{2}=t_{1}+t_{2}$, we get $r_{1}=t_{1}$ and $r_{2}=t_{2}$.

\section{Representations of the Harmonic and the Potential Part}

In this section we present a representation of the harmonic and the potential part of the Riesz decompostion. This representation is inspired by the corresponding result in the context of random walks, confer [Woe00, Woe09]. The validity of such a result is in this sense surprising as the semigroups of Schrödinger operators do not allow for a probabilistic interpretation in the case of non-positive $c$. However, the main idea is to use a ground state transform. This way we get a decomposition to transfer the corresponding decomposition of the random walk context into the context of Schrödinger operators.

In this subsection let $b$ and $c$ be such that $H=H_{b, c, m}$ is non-negative. Hence, $\operatorname{deg}+c>0$ by Lemma 4.1. We define the function $p: X \times X \rightarrow(0, \infty)$ via

$$
p(x, y)=\frac{b(x, y)}{\operatorname{deg}(x)+c(x)}
$$


and the operator $P=P_{b, c}: \mathcal{F} \rightarrow \mathcal{C}(X)$ is defined by

$$
P f(x)=\sum_{y \in X} p(x, y) f(y)
$$

for all $x \in X$. In the setting of random walks, i.e., $c \geq 0$, the function $p$ is called the transition matrix and the operator $P$ is called the transition operator, [Woe09].

We consider the multiplication operator $D=D_{b, c, m}: \mathcal{C}(X) \rightarrow \mathcal{C}(X)$ defined via

$$
D f(x)=\frac{\operatorname{deg}(x)+c(x)}{m(x)} f(x)
$$

which is invertible since deg $+c>0$ by Lemma 4.1. We denote the inverse by $D^{-1}$. Note that $P, D, D^{-1}$ are positivity preserving and we have on $\mathcal{F}$

$$
H=D(I-P) \text {. }
$$

Letting $n=\operatorname{deg}+c$, we readily see that $I-P$ is a Schrödinger operator such that $(I-P)=H_{b, c, n}$. Obviously, for $s \in \mathcal{F}$, we have $H s=0$ (respectively $H s \geq 0$, $H s \leq 0)$ if and only if $(I-P) s \geq 0$ (respectively $(I-P) s \geq 0,(I-P) s \leq 0)$.

In the Riesz decomposition theorem, Theorem 2.4, we have shown that a superharmonic function $s$ with subharmonic minorant decomposes uniquely as

$$
s=G H s+\operatorname{ghm}_{s}
$$

with potential GHs and harmonic part $\operatorname{ghm}_{s}$. Next, we give the main result of this section, an alternative representation of $G H s$ and $\mathrm{ghm}_{s}$. Furthermore, fixing the measure $m$, we denote the operator of multiplication by the function $\mathrm{gm}$ by $M_{g}$. Specifically, we will use the operator

$$
M_{s^{2}} f=s^{2} m f .
$$

Theorem 5.1 (Representation of the Harmonic and the Potential Part). Let $H_{b, c, m}$ be subcritical. If $s>0$ is superharmonic, then

$$
G H s=M_{s^{2}} D G M_{s^{-2}}(I-P) s=\left(M_{s^{2}} D\right) G\left(M_{s^{2}} D\right)^{-1} H s
$$

and

$$
\operatorname{ghm}_{s}=\lim _{n \rightarrow \infty} P^{n} s .
$$

In particular, the limit in the second equality exists.

Note that if $H_{b, c, m}$ is subcritical there always exists $s>0$ superharmonic by the Allegretto-Piepenbrink theorem, see [KPP17, Theorem 4.2].

The basic idea is to prove a corresponding version of the theorem in the case $c \geq 0$. In this case a subcritical operator $H_{b, c, m}$ is called transient. Furthermore, according to [Sch11, Theorem 6], the Green's function of $H=H_{b, c, 1}$ satisfies for $c \geq 0$

$$
G(x, y)=\frac{1}{\operatorname{deg}(x)+c(x)} \sum_{k=0}^{\infty} P^{k} 1_{y}(x)
$$


for all $x, y \in X$.

The proof of the following theorem works along the lines of [Woe09, p. 169].

Lemma 5.2 (Riesz Decomposition for $c \geq 0$ ). Let $b$ be a graph over $X$ and $c \geq 0$ such that $H_{b, c, 1}$ is subcritical and let $s$ be a superharmonic function with subharmonic minorant. Then, the monotone limit

$$
s_{h}=\lim _{n \rightarrow \infty} P^{n} s
$$

exists pointwise and is harmonic, and

$$
s_{p}=D^{-1}\left(s-s_{h}\right)
$$

is a potential with charge $(I-P) s$. In particular, the Riesz decomposition of s with respect to the operator $(I-P)$ is $s=s_{h}+s_{p}$, and with respect to $H$ is $s=s_{h}+D s_{p}$.

Proof. Assume first that $s$ is non-negative. Note that $H s \geq 0$ implies $(I-P) s \geq 0$ and, therefore, $s \geq P s$. Hence, the limit $s_{h}=\lim _{n \rightarrow \infty} P^{n} s$ exists due to monotonicity and the fact that $P$ is positivity preserving. Moreover, we clearly have $P s_{h}=s_{h}$ due to monotone convergence which implies $H s_{h}=0$. Moreover, we have by harmonicity of $s_{h}$, i.e., $(I-P) s_{h}=0$, the representation of the Green's function above and $\left(\sum_{k=0}^{\infty} P^{k}\right)(I-P)=I$, that

$$
G(I-P) s=G(I-P)\left(s-s_{h}\right)=D^{-1}\left(\sum_{k=0}^{\infty} P^{k}\right)(I-P)\left(s-s_{h}\right)=D^{-1}\left(s-s_{h}\right) .
$$

Hence, we obtain for superharmonic $s \geq 0$, that

$$
s=\lim _{n \rightarrow \infty} P^{n} s+D G(I-P) s,
$$

which is the Riesz decomposition of $s$ with respect to the operator $I-P$. Hence, $\lim _{n \rightarrow \infty} P^{n} s$ is the greatest harmonic minorant of $s$ with respect to $I-P$ and therefore also with respect to $H$. But this implies that $D G(I-P) s=G H s$ is the corresponding potential of the decomposition with respect to $H$.

Assume now that $s$ is superharmonic with subharmonic minorant but not necessarily non-negative. Then by Theorem 3.4 the greatest harmonic minorant ghm $_{s}$ exists and $s-\mathrm{ghm}_{s}$ is a non-negative superharmonic function. Applying the first part of the proof yields

$$
\begin{aligned}
s-\operatorname{ghm}_{s} & =\lim _{n \rightarrow \infty} P^{n}\left(s-\operatorname{ghm}_{s}\right)+D G(I-P)\left(s-\operatorname{ghm}_{s}\right) \\
& =\lim _{n \rightarrow \infty} P^{n} s-\operatorname{ghm}_{s}+D G(I-P) s,
\end{aligned}
$$

where the second equality can be justified as follows: First of all $\mathrm{ghm}_{s}$ is harmonic and therefore $\lim _{n \rightarrow \infty} P^{n} \operatorname{ghm}_{s}=\operatorname{ghm}_{s}=P \operatorname{ghm}_{s}$. Secondly, since all other involved terms are finite we conclude that $\lim _{n \rightarrow \infty} P^{n} s$ exists and the equality follows.

Since the Riesz decomposition is unique by Theorem 2.4, we obtain the result. 
With the lemma above we can now deduce the statement of Theorem 5.1 by the virtue of the so called ground state representation.

Proof of Theorem 5.1. Let $s>0$ be superharmonic with respect to $H$. We denote

$$
H_{s}=H_{b, c, s^{-2}}=M_{s^{2}} H \text {. }
$$

Clearly, $H$ and $H_{s}$ share the same (super/sub-)harmonic functions and it is not hard to see that

$$
G_{s}=G M_{s^{2}}^{-1}
$$

is the Green operator of $H_{s}$. Furthermore, we set

$$
D_{s}=M_{s^{2}} D \quad \text { and } \quad P_{s}=P .
$$

Then, $H_{s}=D_{s}\left(I-P_{s}\right)$. One readily sees (confer [KPP17, Section 4.2]) that the corresponding ground state representation $H^{s}=H_{b^{s}, c^{s}, 1}$ of $H_{s}$ with respect to $s$ associated with the graph

$$
b^{s}(x, y)=b(x, y) s(y) s(x), \quad \text { and } \quad c^{s}(x)=s^{-1} H_{s} s(x)=M_{s} H s(x), \quad x, y \in X,
$$

acts on $\mathcal{F}^{s}=s^{-1} \mathcal{F}$ and satisfies

$$
H^{s} f=s^{-1} H_{s}(s f) .
$$

Specifically, $c^{s} \geq 0$. Moreover, a function $u$ is (super/sub-)harmonic for $H_{s}$ if and only if $\left(s^{-1} u\right)$ is (super/sub-) harmonic for $H^{s}$. Hence, we are in a position to apply the previous lemma, Lemma 5.2, to $H^{s}=H_{b^{s}, c^{s}, 1}$.

But before doing so we need to consider the Green operator of $H^{s}$ first which acts on $\mathcal{G}^{s}=s^{-1} \mathcal{G}$ via

$$
G^{s} f=s^{-1} G_{s}(s f)
$$

Moreover, on $\mathcal{F}^{s}$, respectively $\mathcal{C}(X)$ we set

$$
P^{s} f=s^{-1} P_{s}(s f), \quad \text { respectively } \quad D^{s} f=s^{-1} D_{s}(s f) .
$$

The operators $P^{s}$ and $D^{s}$ are the corresponding transition operator and degree matrix of $H^{s}$, i.e., $H^{s}=D^{s}\left(I-P^{s}\right)$.

By assumption the superharmonic function $s$ has a subharmonic minorant $u$ for $H$ and $H_{s}$. Then the constant function $1=s^{-1} s$ is superharmonic with subharmonic minorant $s^{-1} u$ for $H^{s}$. Therefore, we can apply Lemma 5.2 to $1=s^{-1} s$ to get

$$
1=D^{s} G^{s}\left(I-P^{s}\right) 1+\lim _{n \rightarrow \infty}\left(P^{s}\right)^{n} 1
$$

which is equivalent to

$$
s=D_{s} G_{s}\left(I-P_{s}\right) s+\lim _{n \rightarrow \infty} P^{n} s .
$$

Since $1_{h}=\lim _{n \rightarrow \infty}\left(P^{s}\right)^{n} 1$ is harmonic for $H^{s}$, the function

$$
s_{h}=s 1_{h}=s \lim _{n \rightarrow \infty}\left(P^{s}\right)^{n} 1=\lim _{n \rightarrow \infty} P^{n} s
$$


is harmonic for $H_{s}$. Furthermore, according to Lemma 5.2 the function $1_{h}$ is the greatest harmonic minorant of 1 with respect to the operator $I-P^{s}$ and, therefore, with respect to the operator $H^{s}$. Hence, it follows that $s_{h}=s 1_{h}$ is the greatest harmonic minorant of $s$ with respect to $H_{s}$ and $H$ : Indeed if $H u=0$ with $s \geq u \geq s_{h}$, then $H^{s}\left(s^{-1} u\right)=0$ with $1 \geq s^{-1} u \geq s^{-1} s_{h}=1_{h}$. Thus, $s^{-1} u$ is the greatest harmonic minorant of $H^{s}$ and therefore, $u=s 1_{h}$ and $s_{h}=s 1_{h}$ is the greatest harmonic minorant of $H_{s}$ (and $\mathrm{H}$ ). We conclude

$$
\operatorname{ghm}_{s}=s_{h}=\lim _{n \rightarrow \infty} P^{n} s .
$$

By the Riesz decomposition, Theorem 2.4, we infer that the potential part $s_{p}=$ $G_{s} H_{s} s$ of $s$ with respect to $H_{s}$ equals

$$
s_{p}=D_{s} G_{s}\left(I-P_{s}\right) s=D_{s} G_{s} D_{s}^{-1} H_{s} s .
$$

Now plugging in the equalities $H_{s}=H_{b, c, s^{-2}}=M_{s^{2}} H, G_{s}=G M_{s^{2}}^{-1}, D_{s}=M_{s^{2}} D$ and $P_{s}=P$ from the beginning of the proof yields the result.

\section{An Application}

Here we show an application of the first Riesz decomposition which is a Brelot type theorem.

Theorem 6.1. Let $H=H_{b, c, m}$ be subcritical and let $s$ be a non-negative superharmonic function on $X$. Then

$$
H s(x) \leq \inf _{y \in X} \frac{s(y)}{G(y, x)}=\frac{s(x)}{G(x, x)}, \quad x \in X .
$$

Moreover, there is equality if and only if $s$ is a strictly positive multiple of $G 1_{o}$ and $x=o$ or if $s=0$.

Remark 6.2. This theorem has a continuous analogue, see e.g. [Hel09, Theorem 5.7.14], and goes back to Brelot, [Bre44]. In the continuous case one even has equality. In contrast we show that in the discrete setting equality always fails to hold apart from two trivial cases. The failure of the analogy between the discrete and the continuum setting stems from the fact that the discrete Green's function does not have a singularity at the diagonal.

We need the following Harnack Principle.

Lemma 6.3 (Harnack Principle, Lemma 4.6 in [KPP17]). Let $C>0$ and fix some $x \in X$. Assume that we have a sequence of positive superharmonic functions $\left(u_{n}\right)$ such that $C^{-1} \leq u_{n}(x) \leq C$. Then there exits a subsequence $\left(u_{n_{k}}\right)$ that converges pointwise to a strictly positive superharmonic function $u$.

Now, we prove the Brelot type theorem. 
Proof of Theorem 6.1. By the Riesz decomposition theorem, Theorem 2.4, we get for all $y \in X$

$$
s(y)=G H s(y)+\operatorname{ghm}_{s}(y) \geq G(y, x) H s(x) .
$$

Since $G(y, x)>0$ we get the desired inequality.

Claim: For all non-negative superharmonic functions $s$ and $x, y \in X$ we have

$$
\frac{s(y)}{G(x, y)} \geq \frac{s(x)}{G(x, x)}>0 \text {. }
$$

Proof of the claim. Let us fix $x \in X$. Recall that for every finite set $K \subset X$ the inverse $\left(H^{K}\right)^{-1}$ exists on $\mathcal{C}(K)$. Let $\left(K_{n}\right)$ be an increasing exhaustion of $X$ with finite sets with $x \in K_{n}, n \in \mathbb{N}$. The goal is to apply the minimum principle, Theorem 3.2, to

$$
u=\frac{G(x, x)}{s(x)} s-\left(H^{K_{n}}\right)^{-1} 1_{x}
$$

for $K=K_{n} \backslash\{x\}, n \in \mathbb{N}$. Since $\left(H^{K_{n}}\right)^{-1} 1_{x}$ is harmonic on $K_{n} \backslash\{x\}$, we have $H u \geq 0$ on $K_{n} \backslash\{x\}$. Moreover, on $X \backslash K_{n}$, we have $u \geq 0$ and, furthermore, $u(x)=0$ by definition of $u$, i.e., $u \geq 0$ on $X \backslash K_{n}$. Hence, we can apply the minimum principle to $u$ on $K_{n} \backslash\{x\}$ and get that $u \geq 0$ on $X$. Since $\left(H^{K_{n}}\right)^{-1} 1_{x} \nearrow G 1_{x}$ pointwise by [KPP17, Theorem 5.16], we infer, for any $y \in X$

$$
\frac{G(x, x)}{s(x)} s(y)-G(x, y) \geq 0
$$

and, therefore, the claim follows.

The claim proves the equality in the statement of the theorem.

Next, we turn to the characterization of equality. Clearly, we have equality whenever $s$ is a strictly positive multiple of $G 1_{o}$ and $x=o$, or $s=0$.

On the other hand, assume there is equality in $x$. Then,

$$
G(x, x) H s(x)=s(x)=G H s(x)+\operatorname{ghm}_{s}(x)
$$

by the Riesz decompostion, Theorem 2.4, and, therefore,

$$
\sum_{z \in X \backslash\{x\}} G(x, z) H s(z)+\operatorname{ghm}_{s}(x)=0 .
$$

Since all terms involved are non-negative by Theorem 2.4, we infer that they must be equal zero. If ghm $_{s}$ vanishes in $x$ it vanishes everywhere by the the Harnack inequality, Proposition 3.6. This gives that $s$ is a potential with non-negative charge which has to vanish everywhere outside of $x$. This leaves the cases of $s$ being either a strictly positive multiple of $G 1_{x}$ or $s=0$.

Acknowledgements. The authors acknowledge the financial support of the DFG.

The paper is based on the first part of the master's thesis [Fis18]. 


\section{REFERENCES}

[AG01] David H. Armitage and Stephen J. Gardinger. Classical Potential Theory. Springer Monographs in Mathematics. Springer, 1. ed. edition, 2001.

[BB87] Nicu Boboc and Gheoghe Bucur. Order and Convexity in Potential Theory, volume 1344 of Lecture Notes in Mathematics, pages 24-41. Springer, Prague, 1987.

[BGK15] Michel Bonnefont, Sylvain Golénia, and Matthias Keller. Eigenvalue Asymptotics for Schrödinger Operators on Sparse Graphs. Ann. Inst. Fourier (Grenoble), 65(5):1969-1998, 2015.

[BP18] Siegfried Beckus and Yehuda Pinchover. Shnol-type Theorem for the Agmon Ground State. J. Spectr. Theory, to appear.

[Bre44] Marcel Brelot. Sur les ensembles effilés. Bull. Math. Soc. France, 68:12-36, 1944.

[CdVTHT11] Yves Colin de Verdière, Nabila Torki-Hamza, and Françoise Truc. Essential SelfAdjointness for Combinatorial Schrödinger Operators III-Magnetic fields. Ann. Fac. Sci. Toulouse Math. (6), 20(3):599-611, 2011.

[Fis18] Florian Fischer. Riesz Decompositions and Martin Compactification Theory for Schrödinger Operators on Graphs. Master's thesis, Potsdam University, 2018.

[Fit00] Patrick J. Fitzsimmons. Hardy's Inequality for Dirichlet Forms. J. Math. Anal. Appl., 250(2):548-560, 2000.

[FOT10] Masatoshi Fukushima, Yoichi Oshima, and Masayoshi Takeda. Dirichlet Forms and Symmetric Markov Processes, volume 19 of De Gruyter Studies in Mathematics. De Gruyter, 2. ed. edition, 2010.

$\left[\mathrm{GHK}^{+} 15\right]$ Agelos Georgakopoulos, Sebastian Haeseler, Matthias Keller, Daniel Lenz, and Radosław K. Wojciechowski. Graphs of Finite Measure. Journal de Mathématiques Pures et Appliquées, 103(5):1093 - 1131, 2015.

[GKS16] Batu Güneysu, Matthias Keller, and Marcel Schmidt. A Feynman-Kac-Itô Formula for Magnetic Schrödinger Operators on Graphs. Probab. Theory Related Fields, 165(1-2):365-399, 2016.

[GMT14] Batu Güneysu, Ognjen Milatovic, and Françoise Truc. Generalized Schrödinger Semigroups on Infinite Graphs. Potential Anal., 41(2):517-541, 2014.

[Gol14] Sylvain Golénia. Hardy Inequality and Asymptotic Eigenvalue Distribution for Discrete Laplacians. J. Funct. Anal., 266(5):2662-2688, 2014.

[GS11] Sylvain Golénia and Christoph Schumacher. The Problem of Deficiency Indices for Discrete Schrödinger Operators on Locally Finite Graphs. J. Math. Phys., 52(6):063512, 17, 2011.

[Han87] Wolfhard Hansen. Balayage Spaces - A Natural Setting for Potential Theory, volume 1344 of Lecture Notes in Mathematics, pages 98-117. Springer, Prague, 1987.

[Hel73] Lester L. Helms. Einführung in die Potentialtheorie. De Gruyter Lehrbuch. De Gruyter, 1. ed. edition, 1973.

[Hel09] Lester L. Helms. Potential Theory. Universitext. Springer, 1. ed. edition, 2009.

[HK11] Sebastian Haeseler and Matthias Keller. Generalized Solutions and Spectrum for Dirichlet Forms on Graphs, pages 181-199. Springer Basel, Basel, 2011.

[KL12] Matthias Keller and Daniel Lenz. Dirichlet Forms and Stochastic Completeness of Graphs and Subgraphs. Jour. reine angew. Math., 666:189 - 223, 2012.

[KLSW17] Matthias Keller, Daniel Lenz, Marcel Schmidt, and Radosław K. Wojciechowski. Note on Uniformly Transient Graphs. Rev. Mat. Iberoam., 33(3):831-860, 2017.

[KPP17] Matthias Keller, Yehuda Pinchover, and Felix Pogorzelski. Criticality Theory for Schrödinger Operators on Graphs. J. Spectr. Theory, to appear arXiv:1708.09664. 
[KPP18] Matthias Keller, Yehuda Pinchover, and Felix Pogorzelski. Optimal Hardy Inequalities for Schrödinger Operators on Graphs, 2018.

[KR16] Markus Klein and Elke Rosenberger. Agmon Estimates for the Difference of Exact and Approximate Dirichlet Eigenfunctions for Difference Operators. Asymptot. Anal., 97(1-2):61-89, 2016.

[KS17] Evgeny Korotyaev and Natalia Saburova. Schrödinger Operators with Guided Potentials on Periodic Graphs. Proc. Amer. Math. Soc., 145(11):4869-4883, 2017.

[KSK66] John G. Kemeny, J. Laurie Snell, and Anthony W. Knapp. Denumerable Markov Chains. The University Series in Higher Mathematics. Van Nostrand Company, 1. ed. edition, 1966.

[MS68] Gabriel Mokobodzki and Daniel Sibony. Sur une Propriété Characteristique des Cones de Potentiels. C. R. Acad. Sci. Paris, 266:215 - 218, 1968.

[Rie30] Frèdèric Riesz. Sur les Fonctions Subharmoniques et Leur Rapport à la Théorie du Potentiel. Acta Math., 54:321-360, 1930.

[Sch11] Marcel Schmidt. Global Properties of Dirichlet Forms on Discrete Spaces. Diploma thesis, Jena University, 2011.

[Soa94] Paolo M. Soardi. Potential Theory of Infinite Networks, volume 1590 of Lecture Notes in Mathematics. Springer, 1. ed. edition, 1994.

[Tak14] Masayoshi Takeda. Criticality and Subcriticality of Generalized Schrödinger forms. Illinois J. Math., 58(1):251-277, 2014.

[Tak16] Masayoshi Takeda. Criticality for Schrödinger Type Operators Based on Recurrent Symmetric Stable Processes. Trans. Amer. Math. Soc., 368(1):149-167, 2016.

[Woe00] Wolfgang Woess. Random Walks on Infinite Graphs and Groups, volume 138 of Cambridge Tracts in Mathematics. Cambridge University Press, 1. ed. edition, 2000.

[Woe09] Wolfgang Woess. Denumerable Markov Chains. EMS Textbooks in Mathematics. European Mathematical Society, 1. ed. edition, 2009.

Florian Fischer, Institute of Mathematics, University of Potsdam, Germany E-mail address: florifis@uni-potsdam.de

Matthias Keller, Institute of Mathematics, University of Potsdam, Germany E-mail address: matthias.keller@uni-potsdam.de 